\title{
Spin Glasses: Old and New Complexity*
}

\author{
D.L. Stein ${ }^{\dagger}$ \\ Department of Physics and Courant Institute of Mathematical Sciences \\ New York University \\ New York, NY 10003 USA \\ C.M. Newman ${ }^{\ddagger}$ \\ Courant Institute of Mathematical Sciences, New York University \\ New York, NY 10012 USA
}

May 16, 2012

\begin{abstract}
Spin glasses are disordered magnetic systems that exhibit a variety of properties that are characteristic of complex systems. After a brief review of basic spin glass concepts, their use in areas such as computer science, biology, and other fields will be explored. This use and its underlying basis will be termed old complexity. Newer concepts and ideas flowing from more recent studies of spin glasses will then be discussed, leading to a proposal for a kind of new complexity.
\end{abstract}

\section{Introduction}

Spin glasses are disordered magnetic systems in which two nearby localized magnetic moments have a roughly equal probability of interacting either through a ferromagnetic interaction (in which energy is lowered by the moments aligning) or an antiferromagnetic one (energy lowered by antialigning). This can be achieved in several ways. The most common is to substitute, at random locations, a very small concentration (at most a few percent) of a magnetic element, often iron or manganese, inside a nonmagnetic metallic host, such as one of the noble metals (copper, silver, or gold). In these dilute magnetic alloys, spins localized on the impurity atoms polarize the surrounding conduction electron gas in concentric spheres (roughly speaking) of alternating spin polarization (this is known as the RKKY interaction [1]). Depending on the placement of two

${ }^{*}$ This paper is based on a talk given at the Interdisciplinary Symposium on Complex Systems, Halkidiki, Greece, September 19-25, 2011.

${ }_{\dagger}^{\dagger}$ daniel.stein@nyu.edu

${ }^{\ddagger}$ newman@cims.nyu.edu 
nearby magnetic impurities, the conduction-mediated magnetic interaction can therefore be either ferromagnetic or antiferromagnetic.

But there's more than one way to make a spin glass. For example, europium strontium sulfide ( $\mathrm{Eu}_{x} \mathrm{Sr}_{1-x} \mathrm{~S}$, with $x$ typically a few tenths) is also a spin glass. Here the mechanism is different: nearest-neighbor interactions are ferromagnetic, while next-nearest neighbor are antiferromagnetic. Because the magnetic impurity europium is substituted randomly for nonmagnetic strontium, the net effect is again to generate competing ferromagnetic and antiferromagnetic interactions.

The first mechanism leads to a metal, the second an insulator. In addition, spin glasses can be uniaxial (i.e., spins can point only along one axis) or isotropic; they can be crystalline or amorphous. Clearly, spin glasses come in all varieties. What then are the features that they do share?

The most basic is that all possess a disordered ground state configuration: while a typical low-energy atomic configuration of a glass lacks long-range translational order, the spin configuration of a spin glass lacks long-range orientational order; hence the nomenclature.

The signature laboratory features of all spin glasses include 2] a cusp in the low-field ac susceptibility at a frequency-dependent temperature $T_{f}$; a smoothly rounded maximum in specific heat at a temperature slightly above $T_{f}$; localized magnetic moments frozen into random orientations; and very long relaxational or equilibrational timescales.

So why are they interesting?

There are several reasons. From the point of view of physics, spin glasses and other systems with quenched disorder represent a serious gap in our understanding of condensed matter. In more conventional systems, long-range order and useful symmetries enable us to use many of the powerful tools of condensed matter physics and statistical mechanics to establish a conceptual framework to understand their nature and behavior. Such notions include those of Bloch waves, broken symmetry, order parameters, Goldstone modes, topological singularities in order-parameter space, and many others. There are far fewer tools at our disposal for understanding systems with quenched disorder, such as glasses or spin glasses. In this regard, spin glasses are especially interesting because, unlike ordinary glasses, which must be cooled sufficiently rapidly to avoid the crystalline phase, the spin glass has no competing ordered phase. So if a thermodynamic phase transition does exist, then the low temperature phase would truly be an equilibrium condensed disordered phase - a new state of matter.

Note that important qualifier: if a thermodynamic phase transition exists. It is a remarkable fact that, forty years since spin glasses were first identified as such [3], this most basic of all issues remains unresolved. The susceptibility data show a cusp at a temperature identified as $T_{f}$, the "freezing temperature", indicating a phase transition; but the specific heat data show only a rounded maximum, occurring at a temperature typically $10-20 \%$ above $T_{f}$. Many experiments performed since have failed to reconcile these conflicting results. $\mathrm{Nu}-$ merical simulations have yielded some information, indicating the existence of a phase transition in three and higher dimensions [4, 5, 6]. But all we know 
(from theoretical work) for certain is that there is no phase transition in one dimension and that there is one in infinite dimensions. Everything in between remains conjectural.

While this is one of the most fundamental questions we can ask about any condensed matter system, it is only one of many that remain unanswered after decades of experimental, theoretical, and numerical investigations. The problem is that spin glasses do not possess the kinds of symmetries that make accessible the study of crystals, ferromagnets, superconductors, and other homogeneous systems; the absence of these symmetries enormously complicates the analysis of spin glass behavior. The simultaneous presence of both disorder and frustration can lead to new types of broken symmetries, a breakdown of the thermodynamic limit for certain quantities, the emergence of new phenomena such as chaotic temperature dependence, the need for creation of new thermodynamic tools, and other unanticipated features. While it may not be necessary to completely revamp statistical mechanics in order to understand disordered systems, as has sometimes been suggested, it is at least necessary to carefully rethink some deeply held assumptions.

Finally, the study of spin glasses has led to a surprising variety of applications to problems in biology, computer science, economics, and other areas. We'll briefly mention a few of these applications below, but here will simply note that the usefulness of spin glass concepts, enabling them to serve as a bridge to fields outside of physics, is one of the early reasons why spin glasses came to be regarded as relevant to the study of complexity.

\section{Formulation of the Problem}

The modern theory of spin glasses began with the work of Edwards and Anderson (EA) [7, who proposed that the essential physics of spin glasses lay not in the details of their microscopic interactions but rather in the competition between quenched ferromagnetic and antiferromagnetic interactions. They proposed the Hamiltonian

$$
\mathcal{H}_{\mathcal{J}}=-\sum_{<x, y>} J_{x y} \sigma_{x} \sigma_{y}-h \sum_{x} \sigma_{x},
$$

where $x$ is a site in a $d$-dimensional cubic lattice, $\sigma_{x}$ is the spin at site $x, h$ is an external magnetic field, and the first sum is over nearest neighbor pairs of sites only. The couplings $J_{x y}$ are independent random variables chosen from a common distribution (such as Gaussian with mean zero and variance one), and the notation $\mathcal{J}$ indicates a particular realization of the couplings, corresponding physically to a specific spin glass sample. We hereafter restrict ourselves to Ising models, where the only allowed spin values are $\sigma_{x}= \pm 1$.

The disorder is represented by the $J_{x y}$ 's and is quenched; once chosen, they remain fixed for all time, and the spins must adjust as best they can. Physically, this corresponds to the fact that localized magnetic moments in laboratory spin 
glasses (for example, dilute magnetic alloys) are attached to their host impurity atoms, which do not diffuse on laboratory - or indeed, much longer - timescales.

In reality, no laboratory spin glass has an energy function that looks like (10). The great insight behind the EA Hamiltonian is that it is conjectured to be the simplest Hamiltonian that accurately models real spin glasses. The essential ingredient is quenched, randomly placed ferromagnetic and antiferromagnetic couplings between nearby spins. Given this, one can just as well study the spins on a regular lattice; in a real host material, the prime effect of the random placing of magnetic impurities is to generate both ferromagnetic and antiferromagnetic couplings. The restriction in (11) to spin-spin interactions between nearest neighbors only also doesn't occur in real spin glass materials; but the hope is that this again is more of a detail that does not alter its applicability. (Since we're very far from solving even the simple-looking EA Hamiltonian, these assertions have to remain conjectures for now.)

An immediate, and nontrivial, consequence of the competition between ferromagnetic and antiferromagnetic interactions in (11) is the presence of frustration: no spin configuration can simultaneously satisfy all couplings. How does one then find the ground state? Which couplings should be chosen to be unsatisfied? Or could it be that there are possibly many ground states — or at positive temperature, thermodynamic pure states - not connected by any simple symmetry transformation? This is a very intriguing question, and remains one of the central unsolved problems in spin glass research. It is also one of the prime features of spin glasses that has caught the attention and interest of complexity researchers.

Before proceeding further, we emphasize that one can have quenched disorder without frustration (the most well-known example being the Mattis model [8]), and on the flip side, one can have frustration without disorder (for example, a planar antiferromagnet on a triangular lattice). Spin glasses are hard to analyze at least partly because of the joint presence of quenched disorder and frustration - but this also makes them useful as a model system with which to examine certain aspects of complexity, as we'll see.

\section{Mean Field Theory}

The most studied and best understood spin glass model is the infinite-ranged version of the EA Hamiltonian, proposed by Sherrington and Kirkpatrick (SK) 9. For a system of $N$ Ising spins in zero external field, the SK Hamiltonian is given by

$$
\mathcal{H}_{\mathcal{J}, N}=-\frac{1}{\sqrt{N}} \sum_{1 \leq i<j \leq N} J_{i j} \sigma_{i} \sigma_{j}
$$

where the independent, identically distributed couplings $J_{i j}$ are again chosen from a Gaussian distribution with zero mean and variance one. Unlike the EA model, which has $O(N)$ couplings for a system of $N$ spins, the SK model has $O\left(N^{2}\right)$ couplings; this requires a $1 / \sqrt{N}$ rescaling of the coupling magnitudes 
to ensure a sensible thermodynamic limit for free energy per spin and other thermodynamic quantities.

Sherrington and Kirkpatrick showed that their model had an equilibrium phase transition, but their solution for the low-temperature phase was unstable [9. The correct solution for the low-temperature phase of the SK model was found several years later by Parisi 10, who proposed an extraordinary new kind of symmetry breaking, known today as "replica symmetry breaking", or RSB. The essential idea is that the low-temperature phase consists not of a single spin-reversed pair of states, but rather of "infinitely many pure thermodynamic states" [11, not related by any simple symmetry transformations.

But the most striking feature of RSB is not the existence of many nonsymmetry-related thermodynamic states - though this is already very unusual. What really generated a huge amount of interest and excitement was the way that the states were organized; in particular, the joint properties of "non-selfaveraging" of the distribution of spin overlaps between thermodynamic states, and an ultrametric distance relation between triples of pure states [11, 12, 13.

These require some explanation. We already indicated that there are many equilibrium spin glass states, and since all are disordered, there are no easily distinguishable features to categorize them. An alternative is then to see how similar they are to each other, by introducing a spin overlap function $q_{\alpha \beta}$ between two pure states $\alpha$ and $\beta$ :

$$
q_{\alpha \beta}=\frac{1}{N} \sum_{i=1}^{N}\left\langle\sigma_{i}\right\rangle_{\alpha}\left\langle\sigma_{i}\right\rangle_{\beta} .
$$

Although there are many pure thermodynamic states, any spin glass at some fixed temperature and field can be found in only one, and so we need to consider the probability $W_{\alpha}$ that the system will be found in state $\alpha$ (this of course depends on temperature and field, as well as $\mathcal{J}$, but we'll suppress these dependences for ease of notation). $W_{\alpha}$ is usually called the weight of state $\alpha$, and of course $\sum_{\alpha} W_{\alpha}=1$. Consequently, if you choose two spin configurations independently from the Gibbs measure, the probability $P_{\mathcal{J}}(q) d q$ that their spin overlap will be between $q$ and $q+d q$ is given by

$$
P_{\mathcal{J}}(q)=\sum_{\alpha \beta} W_{\alpha} W_{\beta} \delta\left(q-q_{\alpha \beta}\right),
$$

where $\delta$ is the Dirac delta-function and $P_{\mathcal{J}}(q)$ is called the spin overlap density. The subscript $\mathcal{J}$ indicates that the procedure is done for fixed coupling realization.

In the SK spin glass, the overlap density has a complicated form. Lengthy analyses [12, 13, 14, 15, 16] have shown that only a handful of thermodynamic states at low (but nonzero) temperature have weights that aren't extremely small. One must also take into account the self-overlap of each pure state with itself; it was shown that all pure states have the same self-overlap, regardless of coupling realization (as always, aside from a set of measure zero). The fixed 
value of this self-overlap is known as the Edwards-Anderson order parameter $q_{E A}$. Between $-q_{E A}$ and $+q_{E A}$ one has a complicated structure of overlaps between different pure states.

But that's not what's surprising. What's surprising is that it was found that no matter how large $N$ becomes, the positions and weights of all overlaps strictly between $\pm q_{E A}$ vary with coupling realization. (The overlaps $\pm q_{E A}$, on the other hand, are the same for all coupling realizations.)

This may seem problematic: thermodynamics works because different samples behave the same way in the large- $N$ limit. Here's a situation where sampleto-sample fluctuations of an important macroscopic property do not diminish as $N \rightarrow \infty$. This is what is known as non-self-averaging; it represents an important and distinguishing feature of the SK spin glass.

Although non-self-averaging is unusual, it can be reconciled with our understanding of how thermodynamics should behave. The usual, measurable thermodynamic quantities - energy, free energy, magnetization (in nonzero field), and so on - are in fact self-averaging in the spin glass. The spin overlap, though important as a theoretical tool for understanding spin glass order, has no immediate or obvious observable consequences.

What happens if we average the overlap distribution over all coupling realizations? Analytically we have

$$
P(q)=\prod_{1 \leq i<j \leq N} \int d J_{i j} P\left(J_{i j}\right) P_{\mathcal{J}}(q)
$$

also known as the (averaged) Parisi order parameter. Because there are an uncountable number of coupling realizations, and no special overlap value (aside from the self-overlap $q_{E A}$ and its negative) that might either appear in no coupling realization (leading to a gap) or else appear in some positive-measure set of realizations (leading to a spike), $P(q)$ is smooth between the two spikes appearing at $\pm q_{E A}$.

This is strikingly different from anything seen in more conventional homogeneous systems. But there's yet another surprise. Suppose we look at overlaps from triples of states rather than from a single pair. If we do, we find that any three states have overlap relations of a very special kind. This is usually stated in terms of the "distance" between the states, which is just the overlap subtracted from $q_{E A}$, so the more dissimilar two states - i.e., the smaller the overlap - the larger their distance in configuration space. (And of course, a state has zero distance from itself.)

It turns out that the three distances from any three states form the sides of an equilateral or acute isosceles triangle. A space where this distance relation always holds among any three points is known as an ultrametric space. The canonical example of an ultrametric space is a nested (or tree-like, or hierarchical) structure.

All this is much different from anything observed in homogeneous systems. Now, mean-field theory usually provides a reliable description of the low-temperature properties of finite-dimensional models (and becomes exact above some suffi- 
ciently high but still finite dimension); in particular it is often used to reveal the nature of the broken symmetry in more difficult to solve finite-dimensional models. So it was natural to expect that the RSB mean-field picture should similarly describe the nature of ordering in the EA and other short-range spin glass models. This generated a lot of excitement; in particular, could these properties provide a "universality class" for a wide range of disordered systems - in particular laboratory spin glasses and even structural glasses? We'll return to this question shortly, but turn now to what we might call the "old complexity" features of spin glasses.

\section{4 "Old" Complexity}

By the mid-1980's, a number of features of spin glasses brought them to the attention of scientists interested in problems that had come to be known as "complex systems". These spin glass features included their signature properties of possessing both quenched disorder and frustration; and in particular, the ease of precisely formulating these properties in the spin glass context. All complex systems must exhibit these properties in one form or another; certainly a strict homogeneity or rigid ordering (as in a crystal) would preclude any chance of evolving or adapting to changing environments. And without conflicting constraints and requirements, it is difficult to see how anything approaching complexity - with its implied storage and generation of large information content - could develop.

The spin glass feature that has probably done the most to catch the attention and interest of complexity scientists, though, is the presence of many metastable states, that is, states stable to flips of finite numbers of spins. This is often picturesquely characterized by a "rugged energy landscape" (sometimes also used to denote the presence of many pure or ground states, known to occur in the SK model but whose presence remains controversial in more realistic models such as EA). This feature of many "near-optimal" solutions, along with higher-energy (or cost) dynamical traps, is a feature shared by many problems in complexity.

It is also significant that a hierarchical ordering emerges in the state space structure of states in the SK model. This type of structure, or "near-decomposability", was proposed by Simon [17, 18, as a universal feature underlying the architecture of complex systems. The fact that such a structure emerges naturally from the relatively featureless SK Hamiltonian is profoundly surprising. Whether this structure arises in more realistic models, however, is unknown, and its significance for complexity more generally remains unclear.

All of the features discussed above are static, equilibrium properties; and moreover are mostly derived from theoretical constructs. But real spin glasses in the laboratory exhibit nonequilibrium dynamical features that might be equally, if not more, relevant to other complex systems. That is, their dynamical behaviors are highly anomalous [2, including such characteristics as slow relaxation, irreversibility, memory effects, hysteresis, and aging. All of these are features 
shared - in one form or another - by other complex systems.

Many of these features, whether static or dynamical, have lent themselves to the development of new approaches to studying problems in computer science, biology, economics, and elsewhere [16, 19, 20]; the construction of new algorithms and computational schema for hard combinatorial optimization problems; new analytical approaches to finding bounds on costs of near-optimal solutions in NP-complete problems; models for protein folding and conformational dynamics, maturation of the immune response, prebiotic and biological evolution, and neural networks; and new methods of neural-based computation. The "bridge" 21] that spin glasses provided to numerous problems outside of physics and mathematics led to their inclusion in systems of interest to complexity scientists.

\section{5 "New" Complexity?}

Recent work on the structure of short-range spin glasses (which remain poorly understood) suggests that spin glasses may be "complex" in more subtle ways 22, 23. The low-temperature thermodynamic structure of SK spin glasses provides a rich lode of phenomena that has had a significant impact on complexity studies - what we referred to here and in [23] as "old" complexity. But alternative theoretical models [24, 25, 26] and mathematical investigations [27, 28, 29, 30] suggest that short-range spin glasses do not possess mean-field behavior in any finite dimension (so that the $d \rightarrow \infty$ limit is singular) — though the issue remains controversial. But if true, then spin glasses would present a unique statistical mechanical example of such a phenomenon.

Equally importantly, they could help us understand the limits of applicability of analogies between different types of systems. Complexity science thrives on analogy, and the resulting transference of concepts and techniques from one field to another. But true complexity should also bump up against the limits of this program; at some point every complex system has to display unique features of some kind, and at a fundamental level. The concept of universality classes, which has been one of the central and unifying ideas of statistical mechanics, may be less than well suited for complex systems. The (possibly) wildly different behaviors between short-range spin glasses in any dimension and infinite-range spin glasses (which mimic EA models in infinite dimension) - where one would certainly expect similar low-temperature ordering — could be a foreshadowing of this sort of sui generis behavior.

As another example, consider the lack of a straightforward thermodynamic limit for Gibbs states in systems with many competing pure states 31. (as spin glasses are often claimed to possess). A core assumption throughout statistical mechanics is that the thermodynamic limit reveals the bulk properties of large finite systems. But suppose that the low-temperature EA spin glass possesses many pure states; in that case the connection between the thermodynamic limit and the behavior of finite macroscopic systems becomes far less direct. In fact, the difference between the thermodynamic behavior of conventional ordered 
systems and spin glasses would bear a similar relation to the difference between the dynamical behavior of a classical system with a single fixed point and one with a strange attractor.

Spin glasses possess numerous other features that might be relevant in thinking about other complex systems but that haven't yet been explored in this context. Such features include the presence of disorder chaos 32, 33, temperature chaos [26], and stochastic stability [34. Finally, the construction of new mathematical tools like the metastate [28, 30, 35, 36, 37. may prove as useful for the study of complex systems as any of the other new concepts and tools that have arisen from the study of spin glasses.

\section{Acknowledgments}

This work was supported in part by NSF Grants DMS-0604869 and DMS1106316.

\section{References}

[1] N. W. Ashcroft and N. D. Mermin, Solid State Physics. New York: Holt, Rinehart, and Winston, 1976.

[2] K. Binder and A. P. Young, "Spin glasses: experimental facts, theoretical concepts, and open questions," Rev. Mod. Phys., vol. 58, pp. 801-976, 1986.

[3] V. Cannella, J. A. Mydosh, and J. I. Budnick, "Magnetic susceptibility of Au-Fe alloys," J. Appl. Phys., vol. 42, pp. 1689-1690, 1971.

[4] A. T. Ogielski, "Dynamics of three-dimensional spin glasses in thermal equilibrium," Phys. Rev. B, vol. 32, pp. 7384-7398, 1985.

[5] A. T. Ogielski and I. Morgenstern, "Critical behavior of the three-dimensional Ising spin-glass model," Phys. Rev. Lett., vol. 54, pp. 928-931, 1985.

[6] N. Kawashima and A. P. Young, "Phase transition in the three-dimensional $\pm J$ Ising spin glass," Phys. Rev. B, vol. 53, pp. R484-R487, 1996.

[7] S. Edwards and P. W. Anderson, "Theory of spin glasses," J. Phys. F, vol. 5, pp. 965-974, 1975.

[8] D. C. Mattis, "Solvable spin systems with random interactions," Phys. Lett., vol. 56A, pp. 421-422, 1976.

[9] D. Sherrington and S. Kirkpatrick, "Solvable model of a spin glass," Phys. Rev. Lett., vol. 35, pp. 1792-1796, 1975.

[10] G. Parisi, "Infinite number of order parameters for spin-glasses," Phys. Rev. Lett., vol. 43, pp. 1754-1756, 1979.

[11] G. Parisi, "Order parameter for spin-glasses," Phys. Rev. Lett., vol. 50, pp. 19461948, 1983.

[12] M. Mézard, G. Parisi, N. Sourlas, G. Toulouse, and M. Virasoro, "Nature of spin-glass phase," Phys. Rev. Lett., vol. 52, pp. 1156-1159, 1984. 
[13] M. Mézard, G. Parisi, N. Sourlas, G. Toulouse, and M. Virasoro, "Replica symmetry breaking and the nature of the spin-glass phase," J. Phys. (Paris), vol. 45, pp. 843-854, 1984.

[14] B. Derrida and G. Toulouse, "Sample to sample fluctuations in the random energy model," J. Phys. (Paris) Lett., vol. 46, pp. L223-L228, 1985.

[15] M. Mézard, G. Parisi, and M. A. Virasoro, "Random free energies in spin glasses," J. Phys. (Paris) Lett., vol. 46, pp. L217-L222, 1985.

[16] M. Mézard, G. Parisi, and M. A. Virasoro, eds., Spin Glass Theory and Beyond. Singapore: World Scientific, 1987.

[17] H. Simon, "The architecture of complexity," Proc. Am. Phil. Assoc., vol. 106, pp. 467-482, 1962.

[18] H. Simon, "The organization of complex systems," in Hierarchy Theory - The Challenge of Complex Systems (H. H. Pattee, ed.), pp. 3-27, New York: George Braziller, 1973.

[19] D. L. Stein, "Spin glasses," Scientific American, vol. 261, pp. 52-59, 1989.

[20] D. L. Stein, Spin Glasses and Biology. Singapore: World Scientific, 1992.

[21] P. W. Anderson, "Spin glass Hamiltonians: A bridge between biology, statistical mechanics and computer science," in Emerging Syntheses in Science (D. Pines, ed.), pp. 17-20, Redwood City, CA: Addison-Wesley, 1988.

$[22]$ D. L. Stein, "Spin glasses: Still complex after all these years?," in Decoherence and Entropy in Complex Systems (H.-T. Elze, ed.), Berlin: Springer, 2004.

[23] D. L. Stein and C. M. Newman, Spin Glasses and Complexity. Princeton, NJ: Princeton University Press. to appear.

[24] W. L. McMillan, "Scaling theory of Ising spin glasses," J. Phys. C, vol. 17, pp. 3179-3187, 1984.

[25] D. S. Fisher and D. A. Huse, "Ordered phase of short-range Ising spin-glasses," Phys. Rev. Lett., vol. 56, pp. 1601-1604, 1986.

[26] A. J. Bray and M. A. Moore, "Chaotic nature of the spin-glass phase," Phys. Rev. Lett., vol. 58, pp. 57-60, 1987.

[27] C. M. Newman and D. L. Stein, "Non-mean-field behavior of realistic spin glasses," Phys. Rev. Lett., vol. 76, pp. 515-518, 1996.

[28] C. M. Newman and D. L. Stein, "Thermodynamic chaos and the structure of short-range spin glasses," in Mathematics of Spin Glasses and Neural Networks (A. Bovier and P. Picco, eds.), pp. 243-287, Boston: Birkhauser, 1998.

[29] C. M. Newman and D. L. Stein, "Simplicity of state and overlap structure in finite-volume realistic spin glasses," Phys. Rev. E, vol. 57, pp. 1356-1366, 1998.

[30] C. M. Newman and D. L. Stein, "Topical Review: Ordering and broken symmetry in short-ranged spin glasses," J. Phys.: Cond. Mat., vol. 15, pp. R1319 - R1364, 2003.

[31] C. M. Newman and D. L. Stein, "Multiple states and thermodynamic limits in short-ranged Ising spin glass models," Phys. Rev. B, vol. 46, pp. 973-982, 1992.

[32] H. G. Katzgraber and F. Krzakala, "Temperature and disorder chaos in threedimensional Ising spin glasses," Phys. Rev. Lett., vol. 98, p. 017201, 2007. 
[33] S. Chatterjee, "Disorder chaos and multiple valleys in spin glasses," 2009. ArXiv:0907.3381.

[34] M. Aizenman and P. Contucci, "On the stability of the quenched state in mean field spin glass models," J. Stat. Phys., vol. 92, pp. 765-783, 1998.

[35] M. Aizenman and J. Wehr, "Rounding effects of quenched randomness on firstorder phase transitions," Commun. Math. Phys., vol. 130, pp. 489-528, 1990.

[36] C. M. Newman and D. L. Stein, "Spatial inhomogeneity and thermodynamic chaos," Phys. Rev. Lett., vol. 76, pp. 4821-4824, 1996.

[37] C. M. Newman and D. L. Stein, "Metastate approach to thermodynamic chaos," Phys. Rev. E, vol. 55, pp. 5194-5211, 1997. 